\title{
Recognition of enteroinvasive Escherichia coli and Shigella flexneri by dendritic cells: distinct dendritic cell activation states
}

\author{
Ana Carolina Ramos Moreno ${ }^{1 /+}$, Karen Spadari Ferreira ${ }^{2}$, Lucas Gonçalves Ferreira', \\ Sandro Rogério de Almeida', Marina Baquerizo Martinez ${ }^{1}$ \\ ${ }^{1}$ Faculdade de Ciências Farmacêuticas, Universidade de São Paulo, São Paulo, SP, Brasil \\ ${ }^{2}$ Departamento de Doenças Infecciosas, Universidade Federal de São Paulo, São Paulo, SP, Brasil
}

The innate and adaptive immune responses of dendritic cells (DCS) to enteroinvasive Escherichia coli (EIEC) infection were compared with DC responses to Shigella flexneri infection. EIEC triggered DCs to produce interleukin (IL)-10, IL-12 and tumour necrosis factor (TNF)- $\alpha$, whereas S. flexneri induced only the production of TNF- $\alpha$. Unlike S. flexneri, EIEC strongly increased the expression of toll like receptor (TLR)-4 and TLR-5 in DCs and diminished the expression of co-stimulatory molecules that may cooperate to inhibit CD4 ${ }^{+}$T-lymphocyte proliferation. The inflammation elicited by EIEC seems to be related to innate immunity both because of the aforementioned results and because only EIEC were able to stimulate DC transmigration across polarised Caco-2 cell monolayers, a mechanism likely to be associated with the secretion of CC chemokine ligands (CCL)20 and TNF- $\alpha$. Understanding intestinal DC biology is critical to unravelling the infection strategies of EIEC and may aid in the design of treatments for infectious diseases.

Key words: enteroinvasive Escherichia coli - Shigella flexneri - dendritic cells - innate immune response

Enteroinvasive Escherichia coli (EIEC) are closely related to Shigella spp, showing remarkable phenotypic and genotypic similarities. However, the disease induced by EIEC is generally less severe than that induced by Shigella spp (Dupont et al. 1971, 1989, Moreno et al. 2010). This difference in severity may be associated with a lower level of expression of virulence factors by EIEC in the presence of various host cells, such as macrophages and intestinal epithelial cells (Gibotti et al. 2004, Moreno et al. 2009, Bando et al. 2010).

Beyond the virulence factors expressed by certain microorganisms, bacteria are the key players in the complex interactions between invading microbes and host cells and they trigger inflammatory events in the infectious milieu (Ramos et al. 2004, Phalipon \& Sansonetti 2007, Sperandio et al. 2008). The mucosa of the colon is the main site of interaction between EIEC and host cells and in this setting, mucosal dendritic cells (DCs) play a key role in transporting and processing intestinal antigens to Peyer's patches and the mesenteric lymph nodes (Niess \& Reinecker 2006). Specifically, DCs are antigen-presenting cells that establish a link between the innate and adaptive immune responses. Moreover, DCs are the only antigen-presenting cells that migrate to the secondary lymph nodes and present antigens to naïve $\mathrm{T}$ cells (Banchereau et al. 2000). Despite the efficient DC-

Financial support: FAPESP, CNPq

+ Corresponding author: carol@usp.br

Received 24 March 2011

Accepted 1 December 2011 mediated immune response, certain pathogens affect the biological functions of immune cells and subvert the host innate immune response by manipulating the expression of surface molecules and cytokine production, which contribute to adaptive immunity (Sperandio et al. 2008).

Very few studies have addressed how EIEC are recognised by the immune system. Therefore, the lack of information regarding EIEC biology confirms the need to further study the interactions between EIEC and DCs. We hypothesised that there is a difference in host recognition of EIEC and Shigella spp, which may explain the comparatively lower virulence of EIEC.

Here, we report the differential response of a murine intestinal DC-like population, characterised by CD11c expression, to EIEC O124: $\mathrm{H}^{-}$vs. Shigella flexneri M90T. Because pattern recognition receptors on DCs are the likely initiators of the immune response to bacterial infections, we sought to determine how EIEC O124: $\mathrm{H}^{-}$ and $S$. flexneri M90T specifically initiate the toll like receptor (TLR) signalling pathway and the expression of co-stimulatory molecules. These two events lead to the maturation and polarisation of DCs and subsequently drive T-cell activation. We found that murine CD11 ${ }^{+}$ cells expressed large amounts of TLR-4 and TLR-5 after EIEC infection, whereas $S$. flexneri induced only TLR-4 expression (Fig. 1A). We hypothesised that $\mathrm{CD} 11 \mathrm{c}^{+}$ cells respond differently to these two bacterial species because the recognition of EIEC may be dependent on the TLR-4 and TLR-5 signalling pathways, whereas other pathways may be necessary for the recognition of S. flexneri. Other EIEC surface elements may also be involved in EIEC recognition by DCs, such as OmpA, which plays an important role in the adhesion of E. coli to intestinal epithelial cells and triggers the migration of DCs across these cells (Torres et al. 2006). 
Consistent with our findings, Rallabhandi et al. (2008) performed a comparative study of TLR-4 expression in HEK293T cells after activation with different sources of lipopolysaccharide (LPS) and reported that NF- $\mathrm{BB}$ reporter activity was significantly lower in response to $S$. flexneri 2a than to E. coli LPS. Moreover, the recognition of $S$. flexneri was recently linked to TLR-2 and the major outer membrane protein of $S$. flexneri 2a (Pore et al. 2010).

Our study found that only EIEC induced the expression of TLR-5 in CD11c ${ }^{+}$cells. This phenotype was also observed in murine macrophages after infection with EIEC O124: $\mathrm{H}^{-}$, but not when they were infected with $S$. flexneri M90T (data not shown). Cell-surface TLR-5 recognises flagellin, a structural protein that forms a major portion of flagellar filaments (Ramos et al. 2004). Although EIEC are typically immotile, they have been shown to move in modified motility assays conducted at reduced agar concentrations (Andrade et al. 2002). Also in accord with our results, Tominaga et al. (1994) demonstrated that the flagellin gene is cryptic in the genus Shigella. Therefore, our results suggest that future investigations should elucidate the role of the fla- gella in the EIEC invasion process and in EIEC survival within hosts, particularly because flagellin recognition by TLR-5 can trigger proinflammatory and adaptive immune responses. Although motility may represent an evolutionary adaptation important for bacterial survival, this characteristic could also represent a detrimental EIEC phenotype.

The detection of bacterial antigens by DCs initiates intracellular signalling that results in pathogen uptake and processing and the subsequent presentation of antigens to $\mathrm{T}$ cells. This presentation occurs in the context of several stimuli required for naïve T-cell activation, including co-stimulatory signals and cytokine expression (Iwasaki \& Medzhitov 2010). Therefore, to further understand EIEC biology, we analysed the phenotype acquired by CD11c cells challenged with EIEC or S. flexneri, focussing on the expression of surface molecules, cytokine profiles and $\mathrm{T}$ cell activation.

By flow cytometric analysis, we found that CD11 $\mathrm{c}^{+}$ cells infected with EIEC exhibited lower expression of the co-stimulatory molecules CD86 and CD40 compared with CD11c cells infected with $S$. flexneri. Additionally, the expression of major histocompatibility com-
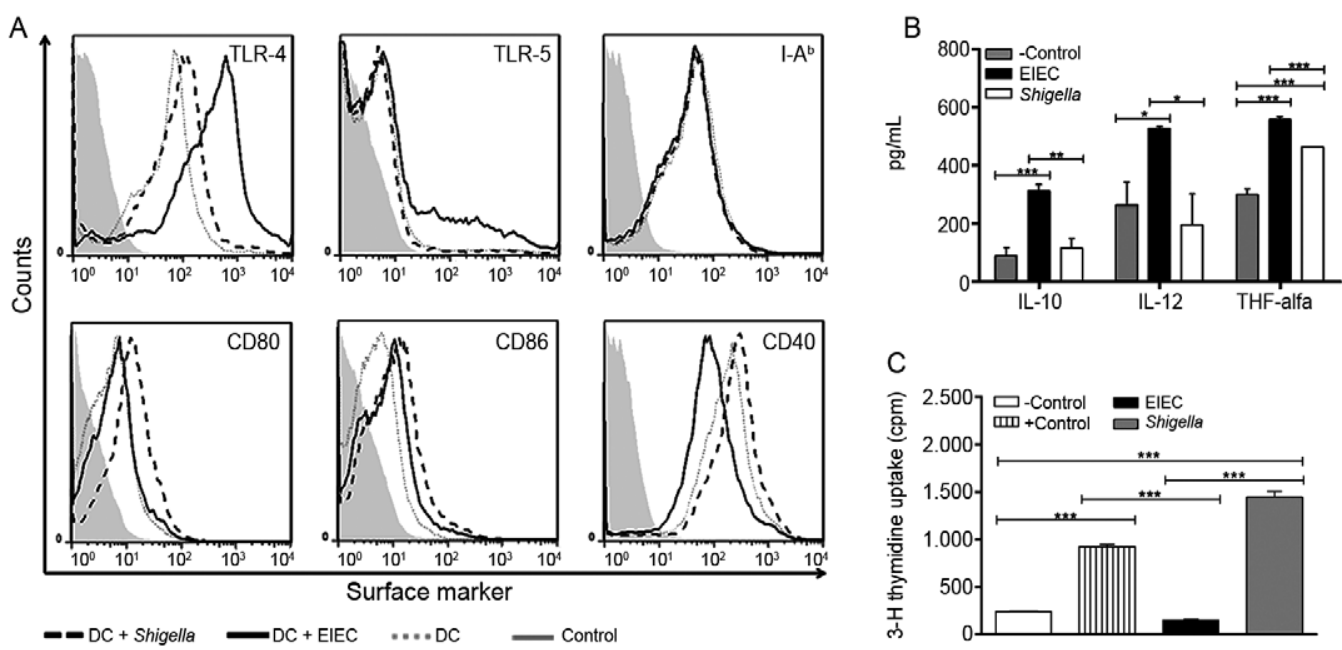

Fig. 1: innate and adaptive immune response of intestinal murine dendritic cells (DCs) infected with enteroinvasive Escherichia coli (EIEC) or Shigella flexneri. Small intestinal segments from naïve C57BL/6 mice (8-12-week-old) were treated with phosphate buffered saline (PBS) containing $10 \%$ foetal bovine serum (FBS), $20 \mathrm{mM} \mathrm{HEPES}, 100 \mathrm{U} / \mathrm{mL}$ penicillin, $100 \mu \mathrm{g} / \mathrm{mL}$ streptomycin, $1 \mathrm{mM}$ sodium pyruvate and $10 \mathrm{mM}$ ethylenediamine tetraacetic acid for $30 \mathrm{~min}$ at $37^{\circ} \mathrm{C}$ with $5 \% \mathrm{CO}_{2}$ to remove epithelial cells. Then, they were washed extensively with ice-cold PBS containing $2 \% \mathrm{FBS}, 100 \mathrm{U} / \mathrm{mL}$ penicillin and $100 \mu \mathrm{g} / \mathrm{mL}$ streptomycin. The tissue was digested in a solution of $0.7 \mu \mathrm{g} / \mathrm{mL}$ collagenase type I (Sigma-Aldrich) and $10 \mathrm{U} / \mathrm{mL}$ DNAse I (Invitrogen) in R10 (RPMI 1.640/10\% FBS plus antibiotics) for 30 min at $37^{\circ} \mathrm{C}$ with $5 \% \mathrm{CO}_{2}$ and continuous stirring. Large particulate matter was removed by passing the cell suspension through a small, loose nylon wool plug. After exposure to plastic for $50 \mathrm{~min}$ to remove macrophages by adherence, intestinal DCs were incubated with MicroBeads (Miltenyi Biotec) conjugated to hamster anti-mouse CD11c monoclonal antibodies. Positively selected DCs contained $>90 \% \mathrm{CD} 11^{+}$cells. These cells also expressed I-A (class $^{\mathrm{b}}$ II major histocompatibility complex), as analysed by flow cytometry. Intestinal DCs were than challenged with EIEC O124:H (FBC124-13) or S. flexneri serotype 5a (M90T) at a ratio of 1:10. A: after $3 \mathrm{~h}$ of infection, DCs were stained with specific monoclonal antibodies and the expression of I-A ${ }^{\mathrm{b}}, \mathrm{B} 7-1$ (CD80), B7-2 (CD86), CD40, toll-like receptor (TLR)-4 and TLR-5 was analysed by flow cytometry using a FACS Canto II (Becton, Dickinson Co). Data are shown as median fluorescence intensity; B: the culture supernatants were collected at $24 \mathrm{~h}$ post-infection to measure interleukin (IL)-10, IL-12p70 and tumour necrosis factor (TNF)- $\alpha$ (pg/mL) by enzyme linked immunosorbent assay (Peprotech); C: bacteria-stimulated DCs (1 h of infection) were co-cultured with naïve mesenteric CD4 ${ }^{+}$T-cells (ratio of 1:3) to investigate the ability of DCs bacterial antigens to lymphocytes. In this experiment, R10 medium was supplemented with $50 \mu \mathrm{g} / \mathrm{mL}$ gentamicin. Methyl- $\left[{ }^{3} \mathrm{H}\right]$ thymidine incorporation was accessed by scintillation counting and the results are expressed as counts per minute (cpm) \pm standard deviation of triplicate cultures. Lymphocytes stimulated with phytohaemagglutinin (PHA) $(20 \mu \mathrm{g} / \mathrm{mL})$ constituted a positive proliferation control. All results are representative of three independent experiments; -control: negative control; +control: positive control; *: $\mathrm{p}<0.05 ; * *: \mathrm{p}<0.01 ; * * * \mathrm{p}<0.001$. 

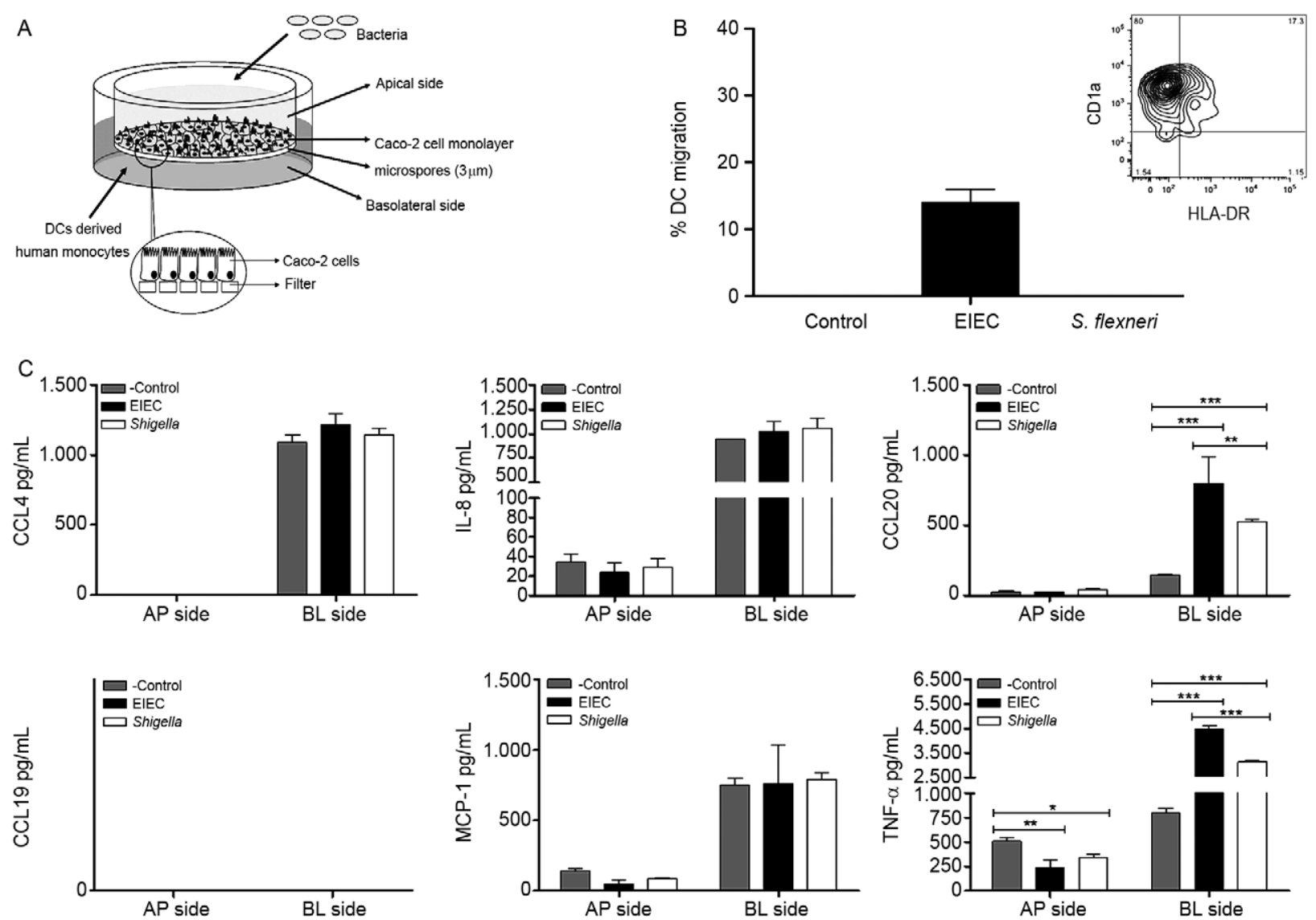

Fig. 2: bacterial-epithelial-dendritic cell (DC) interactions using a transepithelial model. A: Caco-2 cells (ATCC HTB-37) grown on $3 \mu \mathrm{m}$ filter inserts were infected on the apical (AP) surface with $1 \times 10^{6}$ colony-forming unit $/ \mathrm{mL}$ of enteroinvasive Escherichia coli (EIEC) or Shigella flexneri for $2 \mathrm{~h}$ at $37^{\circ} \mathrm{C}$ and $5 \% \mathrm{CO}_{2}$. After this prestimulation time, freshly isolated human DCs $\left(2 \times 10^{5}\right)$ were added to a basolateral (BL) chamber and migration was assessed at $5 \mathrm{~h}$. BL DCs with only medium added to the AP chamber served as a negative control; B: to determine the immune-cell migration, AP and BL supernatants were collected for counting in a haemocytometer. DCs were previously characterised by flow cytometry for the expression of CDla and human leukocyte antigens (HLA)-DR, as indicated in the FACS histogram in the upper right corner of the figure (inset); $\mathrm{C}$ : in addition, AP and BL supernatants from DC migration assays were analysed for cytokine secretion by enzyme linked immunosorbent assay [CC chemokine ligands (CCL)4, CCL19, CCL20, monocyte chemoattractant protein (MCP)-1 and tumour necrosis factor (TNF)- $\alpha$, interleukin (IL)-8 (Peprotech)]. BL of cytokine secretion were detected when polarised Caco-2 cells were incubated with DCs in the absence of bacterial stimulus. The results are shown as means \pm standard deviation for each group and are representative of five independent experiments; -control: negative control; *: $\mathrm{p}<0.05 ; *^{*}: \mathrm{p}<0.01 ; * * * \mathrm{p}<0.001$.

plex II was not altered between EIEC-infected cells and $S$. flexneri-infected cells and only $S$. flexneri induced low CD80 expression (Fig. 1A). During EIEC infection, $\mathrm{CD} 11 \mathrm{c}^{+}$cells also produced higher concentrations of interleukin (IL)-10, IL-12p70 and tumour necrosis factor (TNF)- $\alpha$ (Fig. 1B). Perhaps due to their phenotype, EIEC-infected CD11 $\mathrm{c}^{+}$cells were unable to activate naïve $\mathrm{CD}^{+} \mathrm{T}$-cell proliferation (Fig. 1C). The profile of cells infected with $S$. flexneri was the inverse, exhibiting significant expression of the co-stimulatory molecules CD40, CD80 and CD86 and a greater ability to stimulate T-cell proliferation. Cytokine production, in turn, was only significantly higher in infected cells than in control cells for TNF- $\alpha$. These data suggest that DC-like cells infected with $S$. flexneri have the ability to induce an effective adaptive immune response due to their expression of CD40, CD80 and CD86. However, the inflammation elicited by EIEC may have direct effects on innate immunity during early infection because this strain was able to stimulate the DC-like cells to produce IL-10 and IL-12p70, in addition to expressing TLR-4 and TLR-5. The hyporesponsiveness of cells infected with $S$. flexneri was also reflected in the production of TNF- $\alpha$, IL-6, IL-1 $\beta$ and IL-10 by peripheral blood mononuclear cells. Consistent with our data, cytokine levels were found to be significantly lower in response to $S$. flexneri LPS/ lipid than to E. coli LPS/lipid (Rallabhandi et al. 2008). Recently, Bando et al. (2010) demonstrated that murine macrophages infected with EIEC or S. flexneri produce IL-10 and TNF- $\alpha$, although there is no difference between the levels of cytokines released in response to these two species.

Different antigens and immunisation conditions may lead to responses that vary both quantitatively and qualitatively. In accord with our findings, Sperandio et al. (2008), using a human intestinal infection model, ob- 
served that $S$. flexneri down-regulated innate immunity genes, such as the main DC chemoattractant $c c l 20$. Such down-regulation led to the compromised recruitment of DCs to the lamina propria of infected tissues. In a similar cell-line model (Fig. 2A), we observed that S. flexneri failed to induce DC transmigration across polarised intestinal cell monolayers (Fig. 2B). EIEC, in contrast, were able to induce DC transmigration to the apical side of a transwell, likely resulting in a higher concentration of $\mathrm{CC}$ chemokine ligands (CCL)20 released on the basolateral side (Fig. 2C). Interestingly, flagellin triggers the transient expression of CCL20 in intestinal epithelial cells (Sierro et al. 2001) and induces the constitutive expression of ccl20 in CCR6-producing DCs associated with the Peyer's patch epithelium (Cook et al. 2000). It may be possible to expand this analogy to include the TLR-5-mediated epithelial "education" of DCs, imprinting intestinal mucosal responses to EIEC infection. Our data suggest that intestinal DCs play an important role in the clearance of EIEC infection and that they likely are not as efficient during $S$. flexneri infection.

Clearly, $S$. flexneri adaptively exploits and evades potentially harmful responses of the immune system (Phalipon \& Sansonetti 2007) and this phenomenon could be a key underlying cause of the differential pathogenicity of EIEC and S. flexneri. However, host-pathogen interactions have two components: the virulence and pathogenesis of the bacterium and the inflammatory response of the host's immune system.

In conclusion, we believe that during the inflammation mediated by EIEC-infected DCs, the innate immune response and cell migration are preserved, but the DCs fail to activate naïve T lymphocytes. As a result, the DCs are not fully functioning when they are infected with EIEC. These findings, together with the high production of TNF- $\alpha$ and the potential expression of flagellin by EIEC and of TLR-5 by CD11 ${ }^{+}$cells upon EIEC infection, may represent important differences between EIEC and S. flexneri pathogenicity. Future studies regarding DC function will be needed to further characterise EIEC pathogenicity.

\section{REFERENCES}

Andrade A, Girón J, Amhaz J, Trabulsi L, Martinez M 2002. Expression and characterization of flagella in nonmotile enteroinvasive Escherichia coli isolated from diarrhea cases. Infect Immun 70: 5882-5886.

Banchereau J, Briere F, Caux C, Davoust J, Lebecque S 2000. Immunobiology of dendritic cells. Annu Rev Immunol 18: 767-811.

Bando SY, Moreno ACR, Albuquerque JAT, Amhaz JMK, MoreiraFilho CA, Martinez MB 2010. Expression of bacterial virulence factors and cytokines during in vitro macrophage infection by enteroinvasive Escherichia coli and Shigella flexneri: a comparative study. Mem Insti Oswaldo Cruz 105: 786-791.

Cook DN, Prosser DM, Forster R, Zhang J, Kuklin NA 2000. CCR6 mediates dendritic cell localization, lymphocyte homeostasis and immune responses in mucosal tissue. Immunity 12: 495-503.

Dupont HL, Formal SB, Hornick RB, Snyder MJ, Libonati JP 1971. Pathogenesis of Escherichia coli diarrhea. N Engl J Med 285: 1-9.

Dupont HL, Levine MM, Hornick RB, Formal SB 1989. Inoculum size in shigellosis and implications for expected mode of transmission. J Infect Dis 159: 1126-1128.

Gibotti A, Tanaka TL, Oliveira VR, Taddei CR, Martinez MB 2004. Molecular characterization of enteroinvasive Escherichia coli ipa genes by PCR-RFLP analysis. Braz J Microbiol 35: 74-80.

Iwasaki A, Medzhitov R 2010. Regulation of adaptive immunity by the innate immune system. Science 327: 291-295.

Moreno ACR, Fernandes A, Gomes TDT, Ramos STS, Montemor LPG 2010. Etiology of childhood diarrhea in the Northeast of Brazil: significant emergent diarrheal pathogens. Diagn Microbiol Infect Dis 66: 50-57.

Moreno ACR, Ferreira LG, Martinez MB 2009. Enteroinvasive EScherichia coli vs. Shigella flexneri: how different patterns of gene expression affect virulence. FEMS Microbiol Lett 301: 156-163.

Niess JH, Reinecker HC 2006. Dendritic cells in the recognition of intestinal microbiota. Cell Microbiol 8: 558-564.

Phalipon A, Sansonetti PJ 2007. Shigella's ways of manipulating the host intestinal innate and adaptive immune system: a tool box for survival? Immunol Cell Biol 85: 119-129.

Pore D, Mahata N, Pal A, Chakrabarti MK 2010. 34 kDa MOMP of Shigella flexneri promotes TLR2 mediated macrophage activation with the engagement of NF-kappaB and p38 MAP kinase signaling. Mol Immunol 47: 1739-1746.

Rallabhandi P, Awomoyi A, Thomas KE, Phalipon A, Fujimoto Y 2008. Differential activation of human TLR4 by Escherichia coli and Shigella flexneri 2a lipopolysaccharide: combined effects of lipid A acylation state and TLR4 polymorphisms on signaling. J Immunol 180: 1139-1347.

Ramos HC, Rumbo M, Sirard JC 2004. Bacterial flagellins: mediators of pathogenicity and host immune responses in mucosa. Trends Microbiol 12: 509-517.

Sierro F, Dubois B, Coste A, Kaiserlian D, Kraehenbuhl JP, Sirard JC 2001. Flagellin stimulation of intestinal epithelial cells triggers CCL20-mediated migration of dendritic cells. Proc Nat Acad Sci USA 98: 13722-13727.

Sperandio B, Regnault B, Guo JH, Zhang Z, Stanley SL 2008. Virulent Shigella flexneri subverts the host innate immune response through manipulation of antimicrobial peptide gene expression. J Exp Med 205: 1121-1132.

Tominaga A, Mahmoud MA, Mukaihara T, Enomoto M 1994. Molecular characterization of intact, but cryptic, flagellin genes in the genus Shigella. Mol Microbiol 12: 277-285.

Torres AG, Li Y, Tutt CB, Xin L, Eaves-Pyles T, Soong L 2006. Outer membrane protein A of Escherichia coli O157:H7 stimulates dendritic cell activation. Infect Immun 74: 2676-1685. 